\title{
Nuevas Fronteras del Turismo Rural en Europa - El Proyecto LEADER della Comunidad Europea
}

\section{Andrea Claudia Giangiordano}

RESUMEN: El objetivo de este trabajo consiste precisamente en presentear y describir, a través de la ilustración de un caso deaplicación concreta (el Programa LEADER de la Comunidad Económica Europea), las potencialidades, las ventajasy los riesgos del turismorural y de sus subcategorias, como factor de desarrollo de zonas rurales desłavorecidas. El valor potencial del proyecto en cuestion se encuentra sintetizado en su característica de plena "transferibilidad" a zonas diversas de la realidad europea consintiendo la aplicación de su filosofiay de sus estrategias en otros ámbitos no europeos.

PALABRAS CLAVES: Turismo rural; proyecto "LEADER"; Comunidad Económica Europea.

ABSTRACT: The "Leader Program" from european conmonwealth shows the potentialities, the advantages and the risks of rural tourism as a development factor in rural areas with less economical and social strength. The potential value is demonstrated by its complete adaptation and application in non european areas.

KEY WORDS: Rural tourism; "LEADER" project; european commonwealth.

\footnotetext{
1. Licenciada en Derecho - Universidad de Buenos Aires. Asesora en el campo del turismo regional en el estudio de proyectación "I.S.S." - Idee - Strumenti - Strategie, Marketing, Lanciano (CH). Asesora en la organización de eventos y cursos de formación profesional - Federazione Italiana Pallavolo.

End. para corresp.: Corso Roma, 53 - 66034 - Lanciano (CH). Itália. Tel.: 0039-872-714195 Fax: $0039.872-50628$
} 


\section{Introducción}

La conservación de una agricultura rentable constituye, sin lugar a dudas, un requisito fundamental de toda política de desarrollo rural. Sin embargo, en nuestros dias, la actividad agrícola de por si sola no puede garantizar automaticamente la presencia humana indispensable ni constituir una actividad económica suficientemente diversificada. La caída de los ingresos agrícolas, los peligros de desertación de las zonas sensibles, la preocupación de la población en materia de medio ambiente, y lademanda por parte de los consumidores de productos no uniformesque ofrezcan todas las garantias en cuanto a higiene se refiere, son factores principales que obligan a la reelaboración de los programasde desarrollo de zonas con pocas ventajascomparativas.

La mayor productividad de los sectores secundarios y terciarios y la històrica atracción de las grandes ciudades han ocasionado, hasta hace tiempos recientes, un auténticoéxodo rural. Esta hemorragia ha dejado en las zonas rurakes secuelas tangibles que se traducen en una situación de profundo retraso económico, social y cultural.

Las comunidades rurales han comprendido, sin embargo, que la posibilidad concreta de recuperación y de eventual desarrollo de las zonas en cuestión existe y que no depende ya de medidas exógenas sino de la utilización de los propios recursos locales en actividades económicas competitivas.

En este contexto de crisis de las actividades primarias concebidas en su forma tradicional, el desarrollo rural se construye mediante unaserie de iniciativas locales y territoriales destinadas a diversilicar, partiento siempre de la agricultura, las actividades económicas y a garantizar a la población un nivel cualitativo constante de los servicios y de la asistencia ofreciendo a la juventud las mismas posibilidades en el ámbito de la formación escolar y de la cultura.

El programa LEADER ideadopor la ComunidadEconómicaEuropea (CEE), supo captar las interrelaciones existentesentre las diferentes necesidadesdando una respuesta concreta al problema e identificando, entre otras alternativas, el turismo rural como concreto factor de desarrollo de áreas rurales frágiles.

\section{Metodologia}

El presente trabajo se propone ilustrar, en líneas generales, la filosofia y el campo de acción de una particular iniciativa de la Comunidad Económica Europea llamadaLEADER("Liaisonentre Actions deDéveloppement deL'Economie Rurale") cuyo objetivo es, justamente, analizar las diversas soluciones al problema del desarrollo económico y social de lasáreas desfavorecidas de Europay, especificamente, profundizar una de las soluciones postuladas como potencialmente apta para lograr el citado desarrollo: "el turismo rural".

El estudio parte del análisis documental de la normación vigente a nivel europeo referida a la política agraria comunitaria (PAC)y, en particular, a aquélla que regula la reformafundamental delos FondosEstructurales de la CEEcomo instrumento privilegiado para una política de cohesión económica y social y de solidaridad intercomunitaria (Comisión de las ComunidadesEuropeas, 1993: 7).
Para lograr una mejor comprensión de la temática, se incluyen en el análisis conceptualizaciones, diversos enfoques doctrinarios, yejemplificaciones concretas tratando de construir un esquema general que pueda servir como proyecto operativo viable y "factible" para ser aplicado en realidades diversas a la estudiada.

\section{Turismo Rural en el Ámbito de un Proyecto de Desarrollo Económico de Zonas Marginales}

\section{Proyecto LEADER de la Comunidad Europea}

Desde la realización del Gran Mercado, han surgido numerosos proyectos de colaboración entre los miembros asociados, especialmente en lo que respecta el desarrollo económico-social de las áreas rurales. El proyecto LEADER constituye una especifica iniciativa de la Comunidad Económica Europea llevada a cabo por la Dirección General de la Agricultura (DG VI), cuyo objetivo fundamental es el de identificar las posiblesalternativas para lograr el desarrollo de las zonas rurales menos favorecidas. La iniciativa (91/C 73/14 nace en marzo del 1991 -art. 11 del Reglamento CEE n. 4253/88) proponiéndose para los sucesivos tres años. Dados los resultados positivosobtenidos en el primer período(1991-1993), la Comisión de las Comunidades Europeas decide la continuación del proyecto durante otro período plurienal (19941999). Con esta iniciativa, la Comunidad Europea se propone la promoción de una acciónintegral de desarrollo local de las economías rurales a través de la identificación de alternativas innovadoras y eficases com valor ejemplificativo y viable. Para su concreción há sido constituida una red de Grupos de Acción Locales (GAL) dotados de un importante margen de evaluación y autonomía para la gestión de los recursos a nivel local.

que, de un modo u otro, puedan contribuir al desarrollo de las zonas rurales: administraciones nacionales, colectividades territoriales, grupos de desarrollo local o miembros asociados socioeconómicos. El principal papel de la red es el de intercambiar experiencias y conocimientos. Todos los miembros de la red, se transforman de este modo en "lombres o mujeres recursos".

La intervención se realiza a través de subvenciones globales a favor de organismos competentes designados por el Estado miembro, que se encargan de coordinar la utilización de las mismas por parte de los grupos locales. La filosofiadel proyecto centra su validez en la siguientes ideasfundamentales MacSharry (1992: 2-3):

- no existe desarrollo rural sin una ambición común (compartida por todos los ope

sociedad locales;

- solo act

necesarios de la propia región;

- existe una condición sine qua non: la voluntad de lograr los objetivos a nivel local. 
En base a estos principios básicos, el proyecto se orienta preferentemente hacia acciones que reínan las caracteristicas que se pasan a enumerar:

- integración

- valor demostrativo;

- transferibilidad

- innovación

Sintetizando, cl proyectoLEADER se basa en el concepto básico del "bottom up" (deabajo haciaarriba) (Chanan, 1992: 6-7) quese refiere ala concreta participación de la población local en cada una de las fases del proyecto. Es esta la verdadera novedad y originalidad de la iniciativa que posiula una función integradora y complementaria respecto a la política nacional y regional del desarrollo de las economías nurales y que surge como consecuencia de los escasos resultados del procedimiento inverso "top down" (de arriba hacia abajo), característico de las décadas del ' 60 y'70 (Chassagne, 1992:3).

\section{Turismo Rural como Estrategia de Desarrollo en el Ambito del Proyecto LEADER}

Cuando se habla de desarrollo rural se hace alusión a un concepto territorial y no scctorial. Deallíque, cuando seidentifican losposiblescanales para obtenerlo, no sepierde nunca de vista el ámbito territorial concreto en el que tendrá que llevarse a cabo. Por elloel programa LEADER interesa a 217 zonlas rurales europeas, heterogéneas en cuanto a paisajes, climas, culturas y tradiciones pero homogéneas por su colocación en el sector de las regiones subdesarrolladas, con menor densidad de población (Unidad de Animación LEADER, 1994).

Un territorio rural es un conjunto organizado de personas y de medios cuyo objetivo general consiste en satisfacer una serie de necesidades fisiológicas y psicológicas que le permiten una calidad de vida a su entorno ambiental y a las personas que viven en él, dentro de un contexto armónico y equilibrado.

Los sectorcs en los cuales la acción LEADER de desarrollo interviene son: las producciones agrícolas, el artesanado, las pequeñas y medias empresas, la formación profesional y el turismo rural, específico objeto del presente estudio.

La estrategia general del proyecto se basa en las interrrelaciones de los recursos ambientales, histórico-culturales y de las producciones locales a través de la creación de circuitos productivos-turísticos en grado de presentar nuevas respuestas a la moderna demanda de productos, entre los cuales se cuenta el turismo rural en ambientes ecológicamente equilibrados.

El presente trabajo se concentrara en el estudio de solamente una de las estrategias de desarrollo común postulada en el proyectocomunitariode referencia: el turismo rural.

Muchos proyectos de los grupos de acción local (71 de los 217) han basado la propia estrategia de desarrollo en el turismoruraldadoque entérminoseconómicos representa la sección más importante del programa LEADER (490 millones de ECU) (Unidad de Animación Leader, 1993).

La gestión de los creditos comunitarios es una gestión descentralizada y basada en sus miembros asociados, al igual que para los programas habitualmente financiados por los Fondos Estructurales. Son las regiones los sujetos que reciben la subvención comunitaria que podrán utilizar, como mejor lo estimen conveniente, para:

- asesorar técnicamente en la eleboración de proyectos de desarrollo integrado en un territorio bien definido;

- apoyar programas de inversiones para el desarrollo local, privados o públicos, que demuestren tres características csenciales y nítidamente definidas: la innovación, el valor demostrativo, y la posibilidad de ser transferidos.

El turismo es una palabra clave para los 217 grupos de acción local LEADER porque la varicdad de paisajes y de patrimonios culturales ofrecen a las zonas marginales del proyecto una capacidad para el desarrollo de las distintas formas de turismorural: deportivo, naturalístico, cultural, de "descubrimiento", de relax etc.

Es por ésto que el turismo rural constituye un pilar fundamental de las actividades consideradas prioritarias en el contexto de la redLEADER. La estrategia local de desarrollo del turismo, la comercialización de los productos turísticos, la valorización de los ambientes naturales y la elaboración del llamado producto turistico constituyen temas de enorme interés en la tratación de las temáticas generales del proyecto.

El turismo, por otra parte, esuna actividad de amplísima dimensión internacional en la cual, muchas veces, las zonas consideradas individualmente no disponen de la potencialidad insita de desarrolloque si pueden poseer diversas zonas agregadas en consorcios. Estos últimos pueden ofrecer al "tour operatory" a los distribuidores del turismo rural una amplia gama de destinaciones.

Muchas veces se hace indispensable recurrir a economías de escala para poder permitir la actuación de las acciones necesarias para lograr la promoción, la comunicación y la comercialización de las distintas áreas.

En el sector turístico, al igual que en tantos sectores de la economía de los pueblos, el funcionamiento de las redes y la cooperación entre zonas nurales representan elementos fundamentales para un positivo desarrollo.

Actualmente son 8 las zonas LEADER del sur de Europa que ya cooperan ell el contexto de la red "Via Mediterránea" para promover y comercializar paquetes turísticos culturalcs. Otras 6 zonlas estan organizándose para crear una red informática que permita la gestión de la oferta turística, mientras otras 13 han presentado un proyectode red sobre el tema "Vacaciones insólitas". 
Turismo como Fuerza Motriz del Desarrollo: Efecto Multiplicador

El turismo rural puede desenvolverun precioso rol enlel proceso de desarrollo de las zonas menos favorecidas gracias a su triple función de creador de renta y ocupación, promotor de infrestructuras y medio de intcrcambio y de sinergías entre el ambiente rural y el ambiente urbano.

Pero es su importantc efecto multiplicador sobre las inversiones directas el queloponecomo privilegiado instrument o de desarrollo. Este rol se hace màs incisivo a medida que aumenta el nivel cualitativo y el número de los productos y de los servicios engrado de satisfacer una demanda que, en losúltimos anos, ha demostrado un incremento del $6 \%$ anual, el doble respecto al turismo costero clásico (Calatrava Requena y Ruiz Avilés, 1993:7)

Este programa favorece el mejoramiento de las condiciones para que las empresas agrícolas puedan acarrear ingresos suficicntes y puedan ofrecer posibilidades de trabajo aceptables, siempreen un marco de proteccióndel patrimonio cultural y de la calidad de vida.

Esta calidad de vida cobra una innuencia cada vez mayor en la decisión que toman las nuevas generacionesen el sentidode permanecer en los pueblosy retomar el cultivode las tierras. El programa de renovación de lospueblosabarca al conjunto de aspectos esenciales para las poblaciones nurales, permitiéndoles participar muy de cerca en la organización de su espacio vital, suscitando importantes iniciativas locales. Si a ésto se le agregan las inversiones privadas y publicas derivadas, se evidencia una vez más del efecto multiplicador parael desarrollo.

Ya desde inicios de la década del '50, en muchos países europeos centrales y septent rionales, el turismo rural es considerado como una estrategia prometedora para promover el desarrollo socioeconómico de las zonas desfavorccidas, siendo idóneo para reducir el éxodo de la población y para crear ocupación. Son varios los elementos que permiten lograr este proceso evolutivo: el turismo rural permite la satisfacción de la demanda de espacios propicios a la práctica de una vasta gama de actividades lúdicas, deportivas y de tiempo libre y cultural, satisfaciendo tambiénel creciente interés por el patrimonio natural y la cultura nural de un target ciudadano che, como consecuencia de las características de la vida moderna, se havisto privado de las ventajas de los valores de una vida a pleno contacto con la naturaleza.

Algunossistemas agrícolas extremadamente especializados suscitan, además, un interés turístico particular (ej.: el cultivo de plantas tropicales) dando lugar a la posibilidad de visitas guiadas a las haciendas agricolas con explicaciones sobre los tipos de arboricultura, orígenes, tecnologías utilizadas, mercados, degustaciones de frutas y, sobretodo participaciòn directa a los trabajos campesinos.

En la actualidad, los actores localcs han tomado conciencia de las potencialidades del efecto multiplicador del turismo como medio de creación de rentas complementarias, desarrollo de sinergias o servicios de sostén al mundo rural, a favor de las poblaciones y de los turistas. Estudios realizados en Noruega y en Francia prueban que a los gastos de alojamiento se acompañan distintos tipos de gastos (dos o tres veces mayores) esenciales para el mantenimiento y el desarrollo del comercio y del artesanado local (Calatrava Requena y Ruiz Avilés, 1993:8).

EI caso de Alpujarras, en la provincia de Granada, situada a unos $50 \mathrm{~km}$ de la costa, evidencia las potencialidades del turismo nural de proximidad. En solo diez años, la poblaciónl activa del sector de los servicios aumentó del $25 \%$ al $66 \%$, mientras la población típica mente agrícola descendió pasando del $70 \%$ al $38 \%$. Teniendo en cuenta que la variación demográfica ha sido inexistente, las cifras indican una transferencia masiva del sector activo haciael sectorturístico yactividadescontextuales. El número de los ejercicios es 2,15 veces superior y tal desarrollo contribuye al mantenimiento del artesanato local (tejidos, ceranicas, productos alimentarios tradicionales). En el curso de la ultima década la capacidad de recepción ha aumentado del $250 \%$ y desde hace 5 años se invierten en la región capitales externos. Según el estudio efectuado, el facturado generado en el 1990 por el turismo y las actividades paralelas llega a 2,3 miliones de ECU, una cifra 12 veces superior, en términos corrientes ${ }^{2}$.

\section{Turismo Rural en un Contexto Ambiental}

\section{Turismo Rural: Precisaciones Terminológicas}

Cuando se trata de definir un tipo especial de turismo basado en el contacto directo con la realidad nural son de uso común expresiones tales como turismo rural agroturismo, turismo verde que, com rigor científico, denotam realidades disímiles entre si.

países, corresponden de hecho a realidades extremamente diferentes y complejas. A veces se utiliza la expresión para definir exclusivamente el agroturismo (hospitalidad en haciendasagricolas o factorias), ot ras comprende también el turismo verde, dulce, alternativo, diverso, local, al aire libre ctc.

Es necesario, entonces, precisar la exacta definición terminológicasin olvidar que II

Avilés (1993), expertos en economía y' sociologia agrícola, confirman lo antedicho agrupando las diferentes posiciones doctrinarias en dos principales tendencias:

- aquélla que utiliza como criterio de selección el nivel de los ingresos generados por el sector turístico a benefício directo de la comunidad rural. En este contexto se efectúaı

agroturismo (cada categoria se integra a la anterior como si fueran circulos concéntricos), distinciones que se fundan en el diverso porcentaje de renta derivada del conjunto de la población local o, en el tercer caso, de los agricultores considerados individulamente;

2. Datos estadisticos de la Lirección General VI - Agricultura de la Comisión de las Conunidades Europeas, 1992 
aquélla que toma en consideración todos los elementos que constituyen la oferta. Se hablará, entonces, de turismo nural cuando la cultura nural represente un elemento importante de la oferta. Si se tiene en cuenta la principal actividad especifica de la oferta se hablará de turismo verde, turismogastronómico, ecuestre, náutico, histórico-cultural etc. Es esta ultima la acepción receptada en el presente trabajo que considera el turisimo rural como oportunidad de contacto directo y personalizado con el ambiente nural(físico yhumano)ycomo ocasiónde participación en las actividades, en los usos y en las costumbres de la población local.

\section{Turismo Rural como Oferta}

El turismo rural existe, se desarrolla y gusta porque se diferencia del turismo "industrial" y del turismo "de metas tradicionales", constituyendo un turismo local, un turismo "de pueblo", querido y administrado por los habitantes del pueblo, un turismo de encuentros, y de tiempo compartido (Grolleau, 1993:17).

Se dice que se tratia de un turismo local por cinco razones: porque es de iniciativa local, de gestion local, com provechos locales, caracterizado por paisajes locales y porque valoriza la cultura local

El turismo rural ofrece particularidades que la oferta turistica standard desconoce. El campo, la naturaleza y el aspecto rural no son outra cosa que contextos protectores y tranquilizantes donde el hombre de ciudad puede encontrar una respuesta a su necesidad de reconocimiento afectivo y social. Cuanto màs agresivo es el presente, màs consolador y distensivo se presenta el pasado (Grolleau, 1993:16)

El ritmo de la vida moderna genera la necesidad de probar emociones simples. El "know-how" del turismo nural se funda en la capacidad del campesino de comunicar con aquéllos que han perdido el contacto con la naturaleza, mediante la palabra, las actitudes, el ambiente y las actividades. El mundo rural genera hoy la oferta de un nuevo exotismo, un lujo supremo que podria definirse "lujo de las cosas simples".

El turismo rural es un turismo de pequeña empresa, un turismo en el cual el hombre constituye el elemento central y primario, un tipo de turismo donde los parámetros tradicionales de la oferta son considerados antitéticos con la esencia de la "ruralidad". Por ello, los grandescentros turísticoseficientes ymodernosubicados enambiente nural non cntranen la concepción de "turismo nural" Paradojalmente los "defectos" del turismo nural constituyen la marca que lo identifica. Porello el confort, necesario para el turista, se debe mezclar necesariamente con una atmósfera genuina. Es en esta constante contradicciòn donde se desarrolla el turismo rural para satisfacer las expectativas de una clientela que se delinea cada vez con mayor precisión. Se trata de encontrar el equilibrio entre los valores del pasado yel presente, entre lo que viene demandado y lo que es consumido, entre las expectativas de la ciudad y la realidad del campo. Laautenticidad, lagenuinidad del producto constituye la cualidad màxima de la cultura popularlocal (Charrier, 1991).
Demanda del Turismo Rural

Partiendode la experiencia europea, la crecientedemanda urbana de actividades recreativas en ambientes rurales que se ha registrado en las ultimas décadas ha favorecido la aparición de una oferta turística cada vez más variada a niveles, de zonas locales, específicamente rurales. Se trata, sin embargo, de una demanda extremamente heterogénea: a cada sector de clientela corresponden determinados tiposde actividad turistica que tendrán que ser identificados y adaptados según los casos. En el cuadro del desarrollo integrado del territorio, la colocación de una demanda seleccionada en función de las características de la ofertay de la situación económica local, constituye un factor importante para lograr que las iniciativas actuadas en materia de turismo rural puedan dar los resultados previstos y esperados.

Los estudios realizados sobre el tema indican que gran parte de los amantes del turismo rural, provenientes sobretodo de clases media-alta, atribuyen una gran importancia a los valores y a la identidad cultural local.

Los habitantes de las zonas urbanas se sientem cada vez más atraídos por el campo. Es esta la conclusión que podríamos extraerde la lectura del material existente sobre los progresos del turismo nural en Europa.

Resumiento, el turismo rural constituye hoy un sector económico y social en plena expansión que justifica el creciente interés de un número siempre mayor de operadores, sobretodo promotores y investidores (Guidicini, 1986).

\section{Agroturismo}

El agroturismo se funda cn la agricultura. Actualmente, su importancia es esencial para la cultura rural en cuanto actividad económica. Es, sin dudas, la agricultura la que ha modelado el paisaje rural dándole una comnotaciónespecial. Los amantes del agroturismo demandan productos agrícolas naturales o productos de transformación artesanales. A la agricultura se dcbeel patrimonio einológico (arneses, máquinas, oficios, bodegas etc.).

El agroturismo contribuye a unir los interesesagrícolasy la tutela del ambiente mediante una gestión integral del territorio. A pesar de su importancia creciente, el agroturismo representa todavia una mínima parte del turismo rural (solo el $2 \%$ del facturado) y de la misma agricultura (el $3 \%$ del facturadoagricolo). Son varias las causas queestimulan el crecimiento de este tipo de actividad enEuropa. Por ejemplo, la creciente crisis del sector de la ganadería en las zonas montanas del mediterraneo obliga a los agricultores a diversificarlas actividadesincrementando el valor agregado mediante la venta directa de productos agrícolas y creando riqueza mediante la valorización del patrimonio edificado inutilizado porla empresi.

El agroturismo representa hoy una oportunidad ennumerosas regiones, pero es necesario saberidentificar correctamente las condiciones para el éxito analizando el nivel y la rentabilidad de las inversiones, la profesionalidad de la hospitalidad, la correcta participación en las redes locales y nacionales de comercialización y la 
promoción y el desarrollo de nuevas actividades turísticas en la empresa agrícola (LoSurdo, 1988)

\section{Oferta Agroturistica y su Evolución en Europa}

Lavitalidad del agroturismo como factor de desarrollo puede ser cuantificada teniendo presente los datos que se pasaran a cnunciar. La actividad agroturística inicia en Europa a principios de la década del '80. Desde entonces, el número de las haciendas che ofrecen servicios turísticos se duplicó en Italia, en el Reino Unido y en Francia. Actualmente el número de alojamientos agroturísticos en Europa es superior a 600 mil. En Alemania y en los Países Bajos el porcentaje de haciendas agrícolas que ofrecen una de las formas de alojamiento turístico es del $8 \%$, en Francia del $4 \%$ y en Italia del 2\% (Calatrava Requena y Ruiz Avilés, 1993).

El agroturismo es actualmente una actividad prevalentementefemenina. Las motivaciones declaradas son: el deseo de apertura hacia cl Exterior, la necesidad de entradas suplementarias integrativas y la voluntad de valorizar el patrimonio immobiliario.

El resultado de diversos análisiseconómicosevidencian una notable diferencía entre las haciendas agricolas destinadas a distintas actividades. El alojamiento por si producemedianamente una entrada anual de 2.86() $\mathrm{ECU}$; con servicio restauran la renta varía ent re 3.150 y $5.430 \mathrm{ECU}$; lasfactorías ecuestres y las pensiones ofrecen entradas entre 7.900 y $11 \mathrm{mil}$ ECU. Es claro que lodavia las cifras no pueden compararse a las entradas que olros tipos de turismo, de connotación clásica, producen en ámbito europeo, pero la tendencia al aumento constantepuedeverificarse analizando la oferta agroturística en zonas ya estabilizadas a nivel promocional (en Italia, Toscana).

\section{Condiciones Necesarias para que el Turismo constituya un Factor de Desarrollo de las Zonas Débiles - Riesgos}

Es importante ilustrar que no siempre la elección de diversificar las actividades rurales inclinándose por el turismo ha producido resultados satisfactorios. En muchos casos se ha producido una sobrevaluación de la dimensión turistica en el proceso de desarrollo local lo cual provocó una regresión y, muchas veces la desaparición tolal de la rentabilidad económica de la oferta local. Esta sobrevaluación se tradujo cn un excesivo aumento de estructuras receptivas respecto a la demanda concretamente existente, en la aparición de fenómenos de especulación interna y externa con degrado del ambiente y pérdida del elemento humano y de las relaciones personalizadas, que constituyen el aspecto principal del verdadero turismoverde. A todoésto hay que sumar la falta de instituciones locales apropiadas, la búsqueda prioritaria y desordenada de ganancias, el nivel de formación profesional insuficiente para satisfacer una oferla turistica de calidad, y la falta de planificación.
Se explican, de este modo, la inestabilidad del modelo del desarrollo mediante el turismo y muchos de los resultados negativos en zonas que contaban tcóricamente con grandes potencialidades ambientales y culturales.

Para evitarque talesanomalías puedan tergiversar el iter positivodel proyecto de desarrollo a través del turismo rural, es indispensable identificar cuáles son las condiciones que hacen del turismo un potencial factor de desarrollo local. Se hace entonces necesario:

- mantener un equilibrio optimal entre los sistemas ecológicos, socioeconómicos y culturales de la zona, aún cuando se introduzcan procesos que inducirán al cambio;

- aportar a la población local una fuente de renta suplementaria que permita afrontar la crisis de los sistemas tradicionales de agricultura de la comunidad rural;

- contribuir a la diversificación de las actividades económicas ampliando la oferta de servicios y de productos locales que puedan constituir un ulteriorinterés para la demanda turistica;

- promover el intercambio entre culturas y poblaciones basado en el respeto reciproco como factor de solidaridad y cohesión social;

- favorecer una política coordinada de programación del territorio que permita intensificar la competitividad de las actividades económicas en las zonas más desfavorecidas y que consienta la adquisición de infraestructuras y arneses indispensables para una correcta calidad de la vida.

Es importante recordar que el turismo rural no es un remedio universal y no constituye la solución definitiva a los problemas de marginalidad económica y socialde numerosas zonas rurales. Debe, indefectiblcmente, compaginarse con ot ras actividades en modo armonioso. Por ello el proyecto LEADER no es un programa de desarrollo turístico sino unprograma dedesarrollonural a nivel local. La inversiónenel turismo debe seranalizada en el contexto LEADERcomo unafuerza motriz del desarrollo creadora de actividades en otros sectores. El objetivo de los grupos de acción local es el desarrollo del turismo de calidad, creadorde ocupación y actividadeseconómicas para la población local integrado en el programa de desarrollo global de la zona.

Para garantizar la eficiencia, el programa LEADER prevé subvenciones para:

- valorizar el pat rimonio cultural y natural existente;

- mejorar las infraestrucluras;

- organizar la comunicación y la información luristica definiendo las estrategias de marketing, creando centros de información, publicando depliants informativos, actuando campañas de promoción, organizando manisfestaciones etc.;

- organizar la oferta turística local estableciendo normas cualitativas, creando consorcios y nuevos servicios, abriendo centros de reservas para facilitar el intercambio de demanda;

- crear itinerarios turísticos, circuilos para excursiones a pie, a caballo y en bicicleta;

- preparar a los operadores turísticos. 
En sintesis puede decirse que son cinco los elementos que aparecen decisivos para un buen resultado

- la capacidad de la hospitalidad y el número de las presencias deben ser suficientes para amortiguar las inversiones realizadas:

- los precios tienen que ser calculados en detable y tienen que ser competitivos y rentables;

- la actividad turistica, extremadamente cstacional, debe serintegrada en el calendario de los trabajos agrícolos:

- los gastos operativos y estructurales tienen que ser atentamente controlados; - para poder ofrecer servicios turísticos mís dinámicos es necesario impartir cursos de formación específicos para mejorar la calicuad de las prestaciones ofrecidas.

\section{Conclusiones}

El turismo rural y !n subcategoria del agroturismo constituyen, en el marco europeo communtario un potencial factor de creación de nucva riqueza. Las posibilidades ocupacionales en el sector del turismo rural favorecen el abandono de las actividades agrícolas tradicionales, y el desarrollo de actividades agrícolas innovativas (ej. cultivación y venta de productos biológicos). La participación directa de las instituciones públicas y de los diversos operadores socioeconómicos en la promoción del turismo rural, responde a la necesidad de identificar soluciones que permitan afrontar la crisis económica, los efectos perversosde las reformas a las estructuras agricolas y la destrucción de infraestructuras de base en numerosas zonas nuales.

Enestaóptica, el turismorural permitcuna mejorvalorización delasproducciones tradicionales y del patrimonio immobiliario de la población rural, respetando y tulelando la intangibilidad de áreas y sistemas de vida a beneficio de los habitantes y de lasfuturasgeneraciones y, al mismotiempo, ofrece una válida alternativa a la cada vez más selectiva demanda de propuestas turisticas diversas.

\section{Bibliografia}

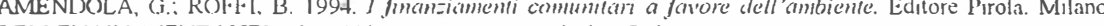
BELLENCIN MENEGHEL, G. 1991. Agroturismo in Italic. Bologna

ismo, una oportunidad para tas zonas rurale

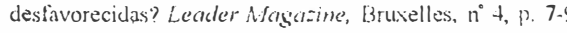

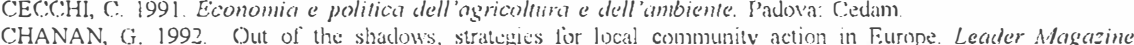
Bruxelles, no 1, p. 6-7.

CHARRIER, J.B. 1991. Geografia dei rapporti cithi-compagna. Milano: F. Angeli

a. Leader Alascizine, Brive

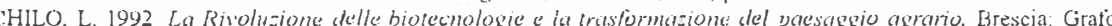

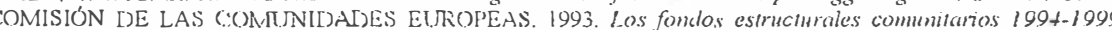
Bruselle
GROLLEAU, H. 1993. Innanzi tutto Temozione!. Lealer Magazine, Bruxelles, n. 4, p. 16-17. GUIDICINI. P. 1986. Il rurale riemergente. Un percorso storico su ipotesi di razionalitá nell agricolo e nella non cittá. Milano: F. Angeli.

Dre Leader Magazine. Bruxelles, in 8. p. 5-9. LO SURDO, Ci. 1988. Agroturismo, risorse, gestione, programm

MACSHARRY, R MAc. Dase Leader "Comercializar un turismo rural de calidad". Bruxelles, p. 15.

Recebido em 7/4/97

Aprovado em 16/6/97 